\title{
MCDR: Mitigating Congestion using Distance based Routing in Wireless Sensor Networks
}

\author{
B M Thippeswamy ${ }^{1}$, Raghavendra $\mathrm{M}^{2}$, Reshma $\mathrm{S}^{2}$, Shaila $\mathrm{K}^{2}$, \\ Venugopal K R ${ }^{2}$, S S Iyengar ${ }^{3}$, L M Patnaik ${ }^{4}$ \\ ${ }^{I}$ Department of Computer Science and Engineering, Jawaharlal Nehru Technological University, Anantpur. \\ ${ }^{2}$ Department of Computer Science and Engineering, University Visvesvaraya College of Engineering, \\ Bangalore University, Bangalore. \\ ${ }^{3}$ Ryder Professor, Florida International University, U S A. \\ ${ }^{4}$ Honorary Professor, Indian Institute of Science, Bangalore, India.
}

\begin{abstract}
The network performance in WSNs is mainly affected by the congestion due to bursty traffic. Congestion can cause large packet drops, increased energy consumption and latency. Different traffic rate control mechanisms have proposed to mitigate congestion and most of these mechanisms are greatly affecting the fidelity requirement of the applications. In this paper, we proposed an algorithm named, Mitigating Congestion using Distance based Routing (MCDR) technique to mitigate congestion. This technique has successfully reduced congestion by scattering the traffic through the nodes which are placed at comparatively minimum distance from the sink and whose Queue Length is below the threshold value. The Residual Energy and Depth are two added parameters to strengthen the scattering decision. Simulation results shows that the network throughput has significantly improved with minimized latency due to the reduction of loops when compared to previous works.
\end{abstract}

Keywords: Congestion, Distance based routing, Wireless Sensor Networks (WSNs), Residual Energy

\section{Introduction}

The scarce resources in WSNs are affected by many factors and degrade the network performance. Congestion is one of the major factors that affect the overall network performance, due to bursty traffic. The applications in WSNs can cause the congestion around the sinks due to centralized traffic pattern. The event driven applications in WSNs produce variable traffic rates in the network and hence it is necessary to maintain fair rate transmission. Rate control and traffic scattering are the most common approaches used to minimize congestion. Although, some of the problems like end-to-end delay, high energy consumption and information processing overheads still exist in the network due to congestion.

Congestion can exist in a network at two different levels viz; the node level and the link level. Node level congestion is due to the buffer overflow and causes the packet loss while link level congestion is due to the contention among the multiple nodes for the transmission medium and degrades the link utilization.. Some of the important and relevant approaches to alleviate congestion are discussed below.

The precedence based data forwarding is one of the approaches that can minimize the congestion. In this approach the high precedence data is forwarded first and low precedence data will be considered next [11]. Precedence based data forwarding can highly penalize the low priority data applications.

Adaptive resource control is one of the congestion control mechanism that dynamically provide the required resource capacity to handle different traffic rate levels [12]. The dynamic provisioning of resource results in managerial overheads in keeping track of the status of the traffic level. The Congestion Detection and Avoidance (CODA) [13] technique is one of the popular approaches to mitigate congestion. It is an upstream mitigation mechanism that uses the queue length of the node and present load on the link to detect congestion. But, the CODA insists on strict transmission policy to acknowledge each packet between the intermediate nodes on the transmission path from source to sink, which increases the end-to-end delay.

The Event to Sink Reliable Transport protocols [14] are developed to achieve event level reliability from source to sink by controlling the congestion in the network. This protocol performs the end-to-end source rate adjustment based on the requirement of reliability level at the base station. There are several mechanisms that performs congestion control by minimizing the hop-by-hop congestion [15][16][1][2][17]. Some of the algorithms detect the congestion based on the rate of channel contention, size of the buffer and hop count [3]. The congestion ratio, packet sender ratio and congestion degree are considered to detect congestion in network [18] [19]. 


\subsection{Motivation:}

Congestion in a wireless sensor network is one of the critical issues that has to be addressed to improve the network throughput and performance. Most of the previous works attempt to mitigate congestion by rate control, adaptive resource provisioning and scattering traffic through multipath and under loaded nodes. Congestion control mechanisms in all these works are developed mainly based on the parameters like queue length, the packet loss ratio, and depth and energy consumption. In our work we have considered the distance of the nodes along with the queue length, residual energy and depth of each node. This mechanism is illustrated by using an example shown in Fig. 1 and Fig. 2.

Let a bursty of traffic be generated at node $\mathrm{A}$ and node $\mathrm{Y}$ and the adjacent node $\mathrm{B}$ is selected to forward the traffic towards the sink through the single path as shown in Fig. 1. It causes congestion at node B and delay the packet transmission towards the sink thereby lowering the network throughput. Hence, it is necessary to mitigate congestion at node $\mathrm{B}$, by choosing multiple paths via lightly loaded nodes. Fig. 2 illustrates the traffic scattering through the multiple paths from node $\mathrm{Y}$ to sink via lightly loaded node $\mathrm{U}$ and from node A to sink via lightly loaded node B. Here, both the node U and node B are placed comparatively at minimum distance to the sink. This mechanism also effectively minimizes the looping problem decreases the Latency and increases the network throughput and minimizes the energy consumption.

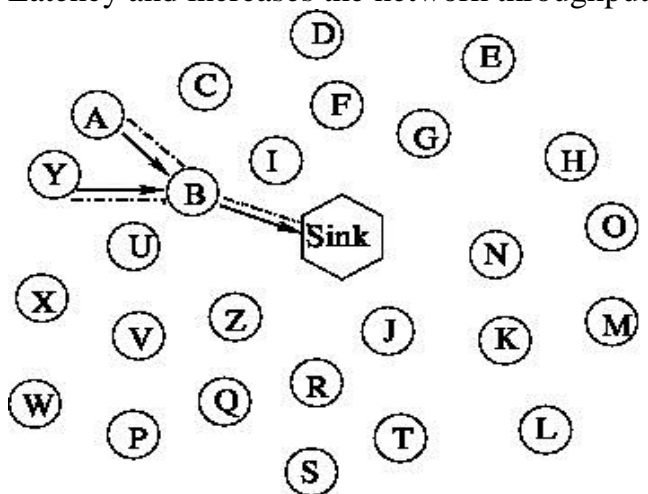

Figure1- Congestion due to single path and Congested node.

\subsection{Contribution:}

The design of our algorithm mainly considers the distance parameter while scattering the traffic through the nodes in the congested area. The queue length, the hop count, residual energy are the additional parameters used along with the distance. The decision of scattering of traffic towards sink based on these parameters effectively reduces energy consumption, looping problems and maximizes the overall network throughput of the network.

\subsection{Organization:}

The rest of the paper is organized as follows. Section 2 presents a brief literature of related works. Section 3 discusses the background work, while section 4 defines the problem and objectives. Section 5 describes the system and mathematical model and proposed algorithm. Section 6 presents the performance evaluation. The concluding remarks are summarized in section 7.

\section{Literature survey}

This section presents a brief summary of related works.

Shigang et al., [4] proposed a congestion avoidance scheme based on light weight buffer management. It prevents data packets from overflowing the buffer space of the intermediate sensors. This approach makes sensors to accustom for optimal forwarding rates without creating the congestion. It is also extended with different MAC protocols, especially for CSMA with implicit ACK, and the 1/k buffer solution prevents hidden terminal from causing congestion. This work illustrates the maintenance of near optimal throughput with minimum buffer size at each sensor and congestion free load balancing for multiple routing paths towards multiple sink. Even though the congestion is effectively alleviated, it affects the fidelity of the applications.

Kang et al., [5] presented a Topology-Aware Resource Adaptation (TARA) algorithm to alleviate congestion. A capacity analysis model is presented that can be used to estimate capacity of different topologies. The careful consideration of type of congestion traffic pattern and network topology is adapted for the use of resource control. This algorithm achieves higher level of data delivery rate and minimized energy consumption when compared to ideal offline resource control algorithm. The resource capacity estimation is a graphic-theoretic 
approach that requires both local knowledge and knowledge about the end-to-end topology. The time overhead is too high for dense networks.

Kumar et al., [6] addressed differentiated data delivery in wireless sensor networks for congested environment. Two algorithms enforce the differentiated routing based on congested area of the network and data priority. The basic protocol Congestion-Aware Routing (CAR) finds the congested zone of the network that is present between high priority data sources and data sink and reserves this area to forward high priority data traffic. Medium Access Control-enhanced CAR (MCAR) is another protocol which is designed to handle the mobility of high priority data sources that cannot be handled by CAR. This scheme gives less importance for low priority traffic, which affects the overall throughput of the network.

Neal et al., [7] implemented relative location estimation algorithms in a WSNs testbed deployed in indoor and outdoor environments. These algorithms are designed, based on a general class of estimation problem known as Cramer-Rao Bound (CRB). The main objective of these algorithms is to show the accuracy with which WSNs can estimate the relative sensor locations. This scheme needs to verify the variance of location estimators due to the non-ergodic nature of shadowing.

Jenn et al., [8] developed a technique to evaluate the quality of path set for multipath load balancing in the presence of wireless interferences. In addition, there are two schemes such as Interference minimized Multipath Routing (I2MR) protocol to increase the throughput by discovering zone-disjoint paths for load balancing. The second one is congestion control scheme that further increases throughput by loading the paths for the load balancing at the highest possible rate supportable. This protocol needs to consider suitably spaced deployment for source-destination pairs and observed the effect of interpath set interferences.

Wang et al., [9] developed upstream congestion control protocol for WSNs, called Priority based Congestion Control Protocol (PCCP). It effectively measures the congestion degree as the ratio of inter-arrival time along over packet service time. Here, the congestion control follows hop-by-hop approach that utilizes the cross layer optimization based on congestion degree and node priority index. PCCP achieves the better congestion control using flexible weighted fairness for both single path and multipath routing. It is also minimizes packet loss rate, the delay and enhanced energy efficiency.

Fengyuan et al., [10] presented the Traffic Aware Dynamic Routing (TADR) protocol to route the packets around the congested areas and divert the overloaded packets along with multiple paths consists of the idle and under loaded nodes. This algorithm uses a hybrid virtual potential field using depth and queue length to divert the packet towards the sink. This scheme improves the fidelity level required by the applications. The TADR does not support delay sensitive and high integrity applications.

Wan et al., [13] proposed a congestion control scheme called, CODA (Congestion Detection and Avoidance). This framework is designed for CSMA based sensors and this congestion control scheme includes three key mechanisms such as receiver based congestion detection, open loop hop-by-hop back pressure and closed loop multi source regulation. The CODA improves the performance of directed diffusion significantly by reducing the average energy tax with minimal fidelity penalty. This work has not been tested for large scale networks.

Yogesh et al., [14] address the notion of sink reliability notion rather than End-to-End reliability. The notion of event to sink reliability is necessary for reliable transport of event features in WSNs. That is because the sink is always interested in collective information of source nodes rather than individual nodes. This idea is implemented through Event Sink Reliable Transport (ESRT) protocol. It achieves the reliable event detection with minimum energy consumption and congestion resolution. The ESRT addresses only the single event in a network, so that it is necessary to check with multiple concurrent events to investigate other possible reliability metrics. It does not support end-to-end reliable data delivery.

Mohammad et al., [15] designed congestion avoidance and fairness algorithms for WSNs. The numerous sensors transmit data to the base station and it increases the probability of packet drops due to congestion close to the base station. This algorithm uses the ratio of the number of downstream and upstream nodes to detect early status of the congestion. Monitoring queue sizes of candidate downstream nodes help to ensure effective load balancing and fairness in this algorithm, hence, it increases the packet delivery ratio and network lifetime. This approach is designed only for the static network topology.

Tian et al., [17] developed a real-time communication protocol for sensor networks. The End-to-End Delay depends on single-hop delay and the distance covered by the packets. SPEED protocol supports a softreal time communication with a desired delivery speed, so that End-to-End Delay is proportional to the distance between the source and destination. It improves the End-to-End Delay and alleviates congestion. Extensive monitoring of information is required for soft real-time communication that drains out energy quickly.

Wan et al., [19] addressed the problem of funnelling effect in many to one multi hop traffic pattern which characterizes the sensor networks communications. This problem can be overcome by exploiting the available wireless and multi radio virtual sinks that can be randomly deployed or placed at selective positions across the networks. These virtual sinks siphon off the data effectively from the congested area in a network. It 
can scale the networks by managing increased traffic demands with minimum impact on application fidelity. The deployment of virtual sinks in a network requires the selection of specific positioning mechanism for effective siphoning off the data for congestion control.

Basu et al., [20] presented a routing paradigm called Potential-Based routing (PB-routing), that is based on gradient search methods to route data packets. The algorithm assigns a scalar potential to network elements, transmits the packets in the direction of maximum positive force and the routing schemes are free from the loops. This algorithm also presents the design of potential function that accounts for traffic condition at a node and the resulting routing algorithm routes around congested areas while preserving the important properties of IP routing mechanisms. It shows significant improvements in end-to-end delay and jitter when compared to previous shortest path routing algorithms. It involves huge management overhead and also explosive to build an exclusive virtual field for every destination in a network.

Hull et al., [21] examined three techniques for mitigating congestion: hop-by-hop flow control, limiting source rate and prioritized Medium Access Control (MAC). The combinations of these three techniques are known as Fusion. Fusion avoids Congestion and improves network efficiency. The rate limiting technique at the each node introduces the monitoring overheads to observe their parent's sending actions to determine the time to generate tokens. This process consumes a large amount of energy.

Kang et al., [22] developed an adaptive resource control scheme to alleviate congestion in resource networks. Here, the dynamic resource provisioning has done based on the capacity level. Congestion alleviation is carried out either by increasing the number of nodes or by providing more routing paths. Resources can also be reduced, when there is no congestion in the network to save energy consumption. It is expensive to control the resource availability and accuracy level required by applications.

Jeongyeup et al., [23] discussed a Rate Control Reliable Transport (RCRT) protocol for constrained sensor nodes in WSNs. It places its congestion control functionality at the sink, so that it enables better aggregate control of traffic and provides flexibility in rate allocation. The RCRT's rates are significantly higher than that of state-of-art in sensor network's congestion control. This protocol is less efficient in co-ordinating the rate allocation across the sinks and it has to improve the convergence time in networks with highly varying Round Trip Time (RTT).

Aris et al., [24] conducted a survey on routing algorithms and identified the requirements and properties that need to be address. The authors addressed possible approaches in the design of efficient routing algorithms for Ad-Hoc sensor networks. Peter et al., [25] analysed and compare reactive single path and multipath routing with load balancing mechanism in ad-hoc networks. The result shows that multipath routing mechanisms create more overhead but provides better performance in congestion and capacity within certain upper bound value of route length. It is found that the performance of multipath routing mechanism decreases above the upper bound value of route length.

Lucian et al., [26] proposed a solution for the betterment of throughput and fairness in wireless networks. This approach has designed with three protocols, namely Biased Geographical Routing (BGR), In network Packet Scatter (IPS) and End-to-end Packet Scatter (EPS). The BGR is one of the multipath routing protocol and IPS, EPS are the two congestion control protocols. The transient congestion and long term congestion alleviates successfully by these protocols and exhibit the simplicity and low communication overhead. This protocol requires large testbed to test EPS and IPS in practice.

Sumit et al., [27] introduced Interference aware Fair Rate Control (IFRC) mechanism to dynamically allocate the fair and efficient transmission rate to each node. It detects the congestion level at each node based on the average queue length and maintained fair and efficient rate. Here the queue overflow is completely avoided and it results in highly effective rate adaptation mechanism. It can be extended easily to support multiple base stations in a network. The end-to-end reliability that is required for the complete validation and analysis of all parameters of IFRC, has not completely provided by this protocol.

Yaghmaee et al., [28] presented a priority based rate control mechanism for congestion control and service differentiation in Wireless Multi Media Sensor Networks (WMSNs). This protocol clearly differentiates the high priority real time traffic from low priority non-real time traffic. It regulates the source traffic rate based on the status of congestion in the upstream nodes and priority of each traffic source. This protocol provides low packet loss probability, low queuing delay and guaranteed bandwidth for high priority real time traffic. The low priority traffic sources suffers from high latency and low reliability.

In the proposed protocol, we have devised an efficient method of congestion control mechanism that mainly considers the distance parameter along with the queue length, depth and residual energy of each node when scattering the traffic towards the sink at the point of congestion. This mechanism minimizes the distance between the source nodes and the sink, looping problem and maximizes the throughput of the network. 


\section{Background}

Most of the existing congestion control protocols mitigate congestion by controlling the traffic rate at the node level. The traffic rate control scheme always minimizes the throughput and brings down the fidelity level required by the application. In order to overcome this problem, the Traffic Aware Dynamic Routing (TADR) protocol is designed to route the packets around the congested areas and divert the overloaded packets along with multiple paths consisting of the idle and under loaded nodes [10]. This algorithm uses a hybrid virtual potential field using depth and queue length to divert the packet towards the sink. The TADR does not support delay sensitive and high integrity applications due to high End-to-End Delay.

Our work mitigates congestion by scattering the traffic, based on the parameters such as the distance, the normalized queue length, depth and the residual energy. The proposed algorithm has effectively increases the throughput and minimizes energy consumption. The end-to-end delay is drastically reduced due to elimination loops.

\section{Problem definition}

In a given wireless sensor network of $N$ randomly deployed nodes, the bursty traffic causes congestion due to high data rate applications. Congestion is mitigated by scattering the packets through the node $N_{i}$, where the value of $i$ varies from 1 to $n$ on the path followed by the packets to the sink. The nodes are selected, based on the status of the queue length $Q_{l}$, depth $d$, the residual energy $R_{e}$ and the distance $D$ to the sink. The main objectives of our research work are:

(1) To improve the network throughput by implementing distance based congestion control.

(2) Reduction of the loops to minimize the Latency.

Assumptions:

(1) Initially all the sensor nodes have equal residual energy and queue length is zero.

(2) The sink consists of highest energy and located at the centre of the network.

\section{System and mathematical model}

The proposed model for Mitigating Congestion using Distance based Routing (MCDR) protocol is illustrated as shown in the Fig. 3.

The system architecture is divided into six different phases. The first phase involves the monitoring of event generation and traffic status. If the nodes are found to be congested, it is necessary to scatter the traffic/packets through the lightly loaded nodes along the multiple paths. This process is carried out in second phase. The third

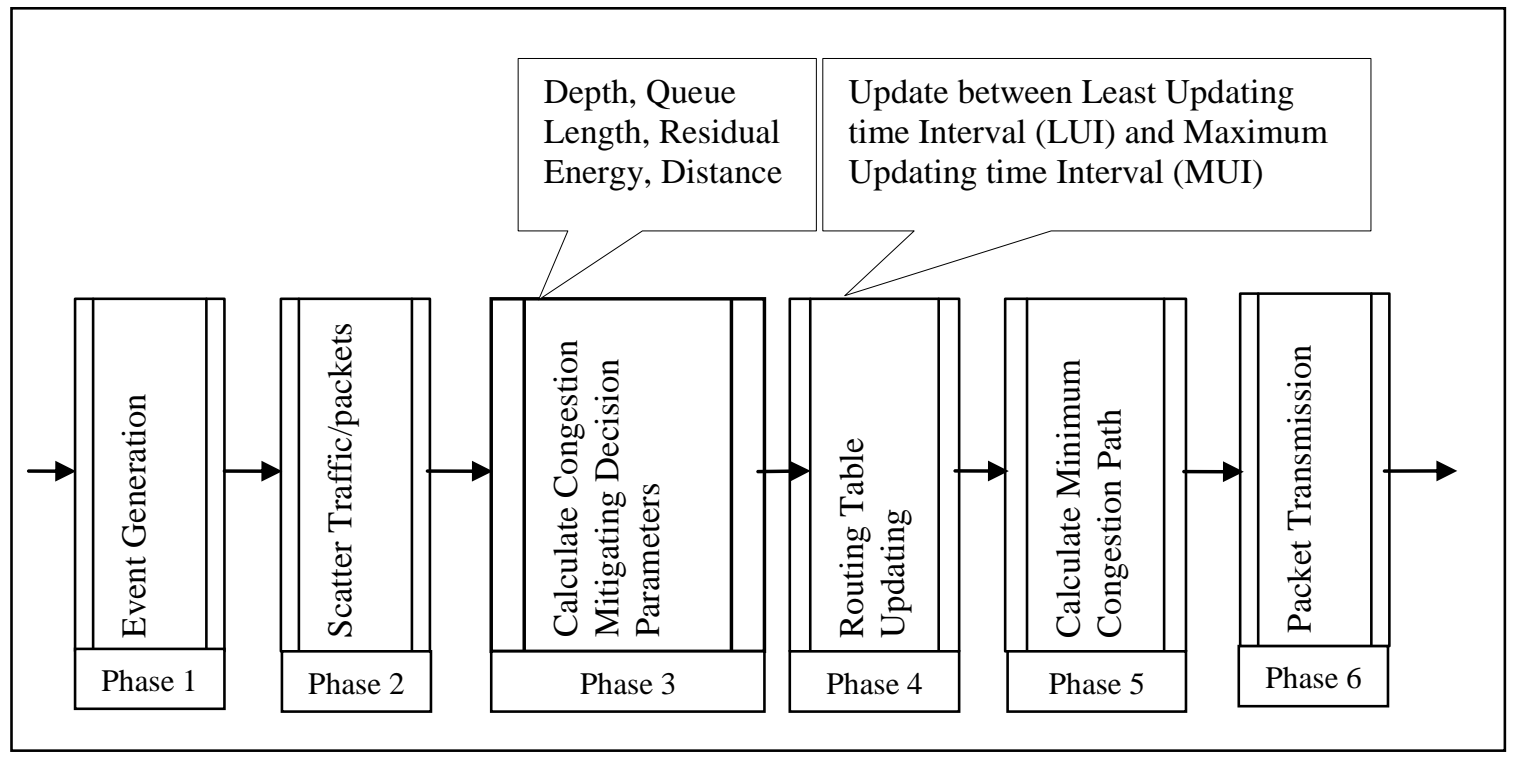

Figure 3 - Proposed model for MCDR protocol

phase involves the calculation of congestion mitigating decision parameters such as depth, queue length, residual energy and the distance to select an appropriate neighbour node. The fourth phase generates the updated routing table values between the Maximum Updating Interval (MUI) and Least Updating Interval (LUI). The fifth phase involves the computation of minimum congestion path based on the congestion mitigating decision parameters. The sixth phase represents the packet transmission towards the sink. 


\subsection{Congestion mitigating decision parameters}

This phase involves the calculation of congestion mitigating decision parameters, such as depth, residual energy, queue length and distance to the sink for each node. These parameters are explained below.

\subsubsection{Depth}

The depth, $d$ of a node is defined as the total number of hop counts between itself and the sink. Initially, the sink initializes its own depth, $d$ as $d=0$, and broadcasts this value to its immediate neighbour nodes. Then, the neighbour nodes updates its depth as, $d=d+1$. This process is repeated until all the nodes compute their depth $d$ to the sink.

\subsubsection{Residual Energy}

The Residual Energy, is defined as the energy retained in each node after the previous receiving and transmission process. The Residual Energy $R_{e}$ can be computed as:

$$
R_{e}=I_{e}-\left(E_{t}+E_{r}\right) \text {, }
$$

where, $I_{\varepsilon}$ is the Initial Energy, $E_{t}$ and $E_{r}$ are the energy required for Transmission and Receiving respectively. The energy for transmission $E_{t}$ is the energy required to transmit each packet and computed as:

$$
E_{\mathrm{t}}=P_{\mathrm{t}}+T_{\mathrm{t}}
$$

where, $P_{t}$ is the number of packets transmitted and $T_{t}$ is the time required to transmit each packet. The Receiving Energy $R_{\mathrm{e}}$ is the energy required to receive each packet. This can be computed as:

$$
E_{r}=P_{r}+T_{t}
$$

where, $P_{\tilde{r}}$ is the number of packets received, $T_{t}$ is the time required to receive each packet.

\subsubsection{Queue length}

The Queue Length, $Q_{l}$ is defined as the ratio of number of packets in the buffer to the maximum buffer size of the node. It can be calculated as

$$
Q_{l}(i)=\frac{N_{p}}{B_{s}(i)}
$$

where $Q_{l}(i)$ is the Queue Length of node $i, N_{p}$ is the number of packets in the buffer, $B_{s}(i)$ is the maximum buffer size of node $i$.

\subsubsection{Distance}

The Distance between the nodes to the sink can be calculated using Euclidean distance formula. The distance $D(i, j)$ is represented as:

$$
D(i, j)=\sqrt{\left(x_{1}-x_{2}\right)^{2}+\left(y_{1}-y_{2}\right)^{2}}
$$

where $D(i, j)$ is the distance between the node $i$ and sink node $j,\left(x_{1}, x_{2}\right)$ is the $x$ co-ordinates of node $i$ and sink node $j$ and $\left(y_{1}, y_{2}\right)$ is the $y$ co-ordinates of node $i$ and sink node $j$.

\subsection{Routing table updation}

In the network, every node consists of the routing table, which stores the updated values of congestion mitigation decision parameters. The routing table updation is carried out four different events. In the first event, the normal routing table updation is done between the Least Updating time Interval (LUI) and Maximum Updating time Interval (MUI). Routing table updation occurs at the expiry of both MUI and LUI(second event). The third event involves routing table updation, when depth changes and LUI expires. The fourth event updates routing table when the queue length exceeds the queue threshold value and LUI expires. The complete routing table updation algorithm is presented in Table I. 
Table I. Routing Table Updation Algorithm

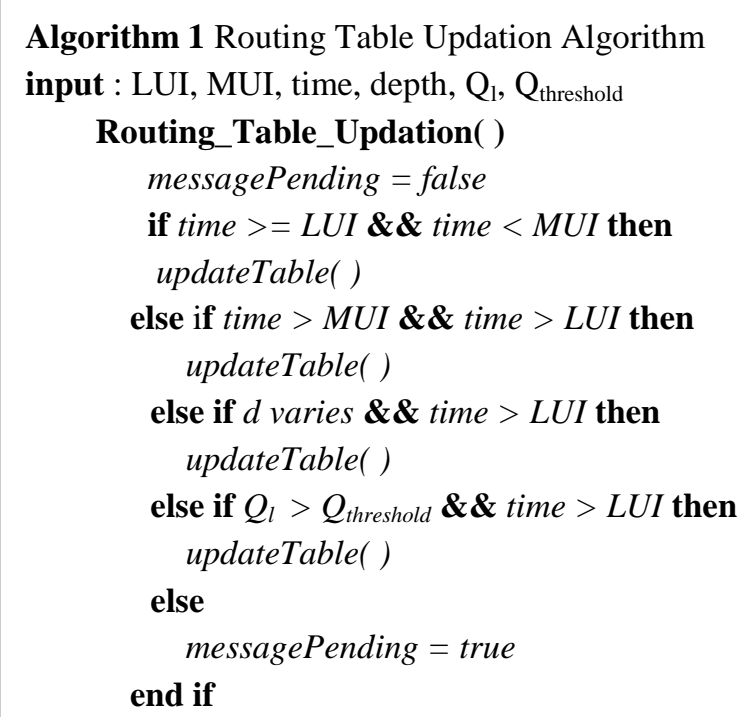

\subsection{Computation of minimum congestion path}

The fifth phase computes the minimum congestion path based on the values of congestion mitigating decision parameters. This computation is based on the updated values of the routing table that enables to select the feasible neighbour with the queue length below the threshold value, maximum residual energy, minimum depth and minimum distance from the sink. If these conditions are not met, the next neighbour node is selected. This process is repeated until the minimum congestion path towards the sink is established. The algorithm is given in Table II.

Table II. Mitigating Congestion using Distance based Routing (MCDR) Algorithm

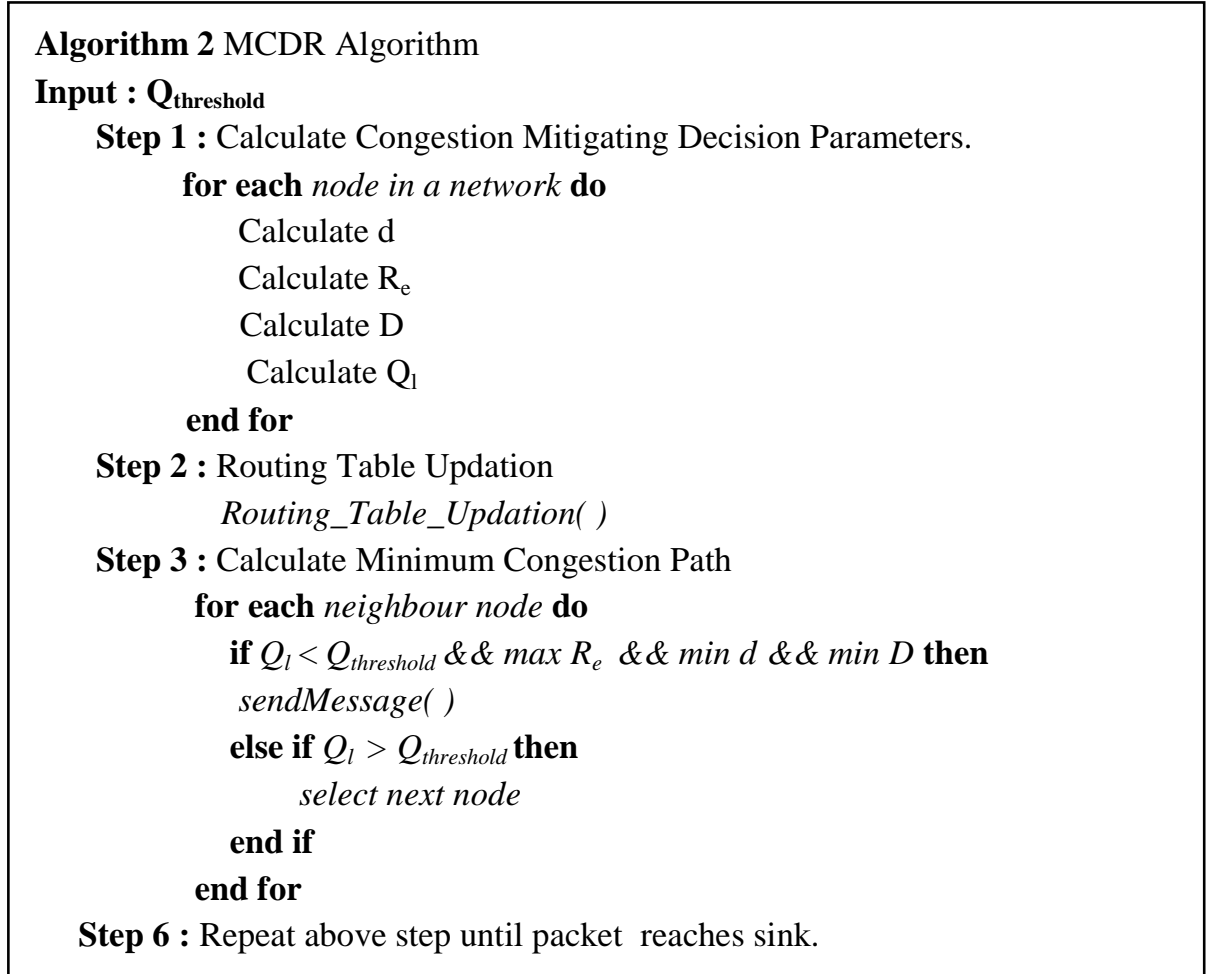




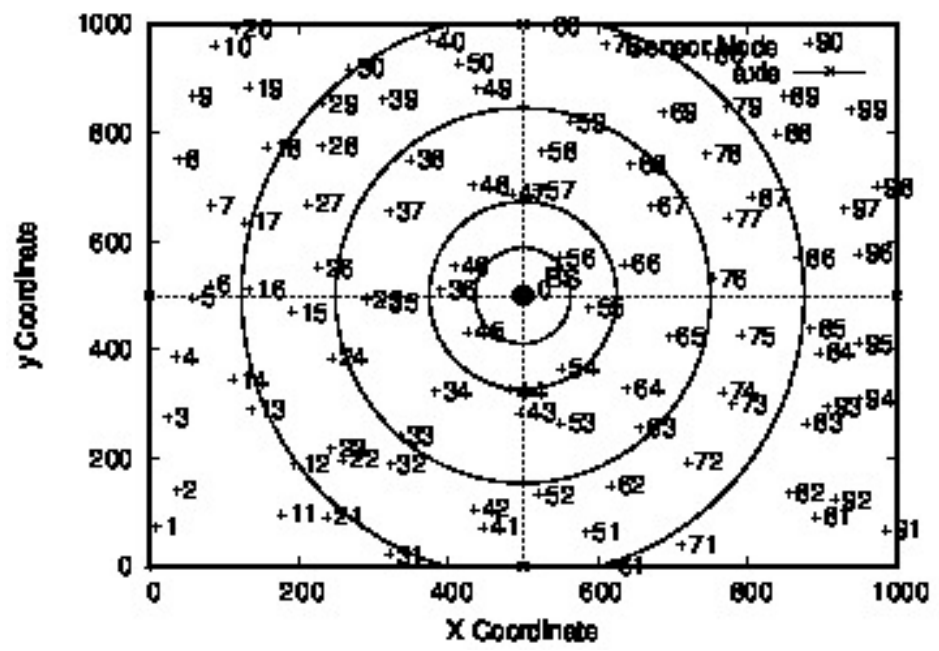

\subsection{Performance metrics}

Figure 4 - Sensor Node Distribution

The following performance metrics are used to analyse the MCDR algorithm.

(a) Network Throughput Rate (NTR): It is defined as the number of packets received per unit of time by the sink from different source nodes.

(b) Packet Delivery Ratio (PDR): It is the ratio of actual packet received by the sink to the total number of packets sent from the source.

(c) Latency: It is the total time required to transmit each packet from the source to the destination.

(d) Network Congestion Rate (NCR): It is defined as the average number of packets queued up at each node per unit of time in a network.

\subsection{Performance analysis}

Table III depicts the Network Size, the Number of nodes deployed, Node Distribution, Initial Energy, Location and Simulation time.

Table IV shows the Network Throughput Rate for the proposed protocol MCDR in comparison with TADR. Fig. 5 illustrates the comparative value of Network Throughput with our protocol MCDR and TADR. This graph illustrates the throughput level of two protocols, MCDR and TADR. MCDR exhibit the better throughput rate for both normal and bursty traffic rate. It is observed that the time between 0 to $4 \mathrm{~s}$ and 7 to $12 \mathrm{~s}$ in normal situations and between 4 to $7 \mathrm{~s}$ and 12 to $14 \mathrm{~s}$ of bursty traffic, the MCDR shows high throughput rate compared to TADR.

Table III -Simulation Parameters

\begin{tabular}{|l|l|}
\hline Parameter & Values \\
\hline Network Size & $1000 \mathrm{~m} * 1000 \mathrm{~m}$ \\
\hline Number of nodes & 100 \\
\hline Node Distribution & Random \\
\hline Initial Energy & $10 \mathrm{~J}$ \\
\hline Data Packet Size & 1024 \\
\hline Sink Location & $500 \mathrm{~m} * 500 \mathrm{~m}$ \\
\hline Simulation time & $2000 \mathrm{~s}$ \\
\hline
\end{tabular}

Table IV - Network Throughput Rate

\begin{tabular}{|l|l|l|l|}
\hline \multirow{2}{*}{$\begin{array}{l}\text { Simulation } \\
\text { Time }\end{array}$} & \multicolumn{4}{l}{$\begin{array}{l}\text { Network } \\
\text { Rate }\end{array}$} \\
\cline { 2 - 4 } & Input & MCDR & TADR \\
\hline 0 & 50 & 45 & 30 \\
\hline 1 & 50 & 45 & 31 \\
\hline 2 & 50 & 41 & 39 \\
\hline 3 & 50 & 42 & 33 \\
\hline 4 & 50 & 46 & 32 \\
\hline 5 & 490 & 368 & 259 \\
\hline 6 & 50 & 383 & 265 \\
\hline 7 & 50 & 44 & 34 \\
\hline 8 & 50 & 42 & 30 \\
\hline 9 & 50 & 44 & 39 \\
\hline 10 & 50 & 43 & 34 \\
\hline 11 & 50 & 48 & 36 \\
\hline 12 & 50 & 41 & 36 \\
\hline 13 & 200 & 123 & 92 \\
\hline 14 & 200 & 123 & 98 \\
\hline
\end{tabular}




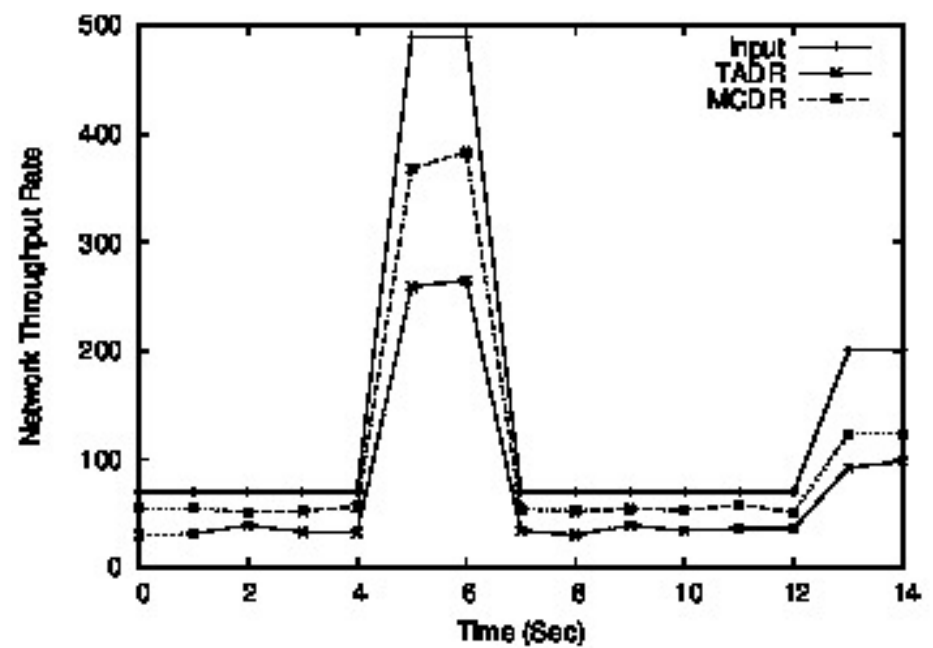

Figure 5 - Network Throughput Rate

This is due to minimized packet drops on account of the balanced Queue Length at each node in the network. The balanced queue length is achieved by the considering the distance of each node to the sink while scattering the traffic towards the sink. It is observed that the network throughput has increased by $62.5 \%$ over the earlier protocol TADR.

Table V shows the packet delivery rate for both at high load and low load condition in the network for MCDR and TADR. Fig. 6 and Fig. 7 illustrate the graphical representation of these values. It is observed in Fig. 6 that both MCDR and TADR shows almost same packet delivery rate when network generates lower number of packets (between 1 to $4 \mathrm{~s}$ ). MCDR exhibits higher packet delivery rate when compared to TADR while the network generates higher number of packets.

It is observed that in Fig. 7 that the packet delivery rate for TADR starts from lower packet delivery rate and increases with minimum value over the time ( 1 to 12s) and does not exceed more than 32 packets in average per second. Whereas, MCDR starts with comparatively higher packet delivery rate and increases linearly over the time ( 1 to $12 \mathrm{~s}$ ) and delivers 82 packets in average per second. Thus, MCDR exhibits better Packet Delivery Rate at both high and low load conditions. It is about $46.5 \%$ higher for high load condition and $37.5 \%$ higher for low-

Table V - Comparison values of Packet Delivery Rate for Low and High Load and Latency

\begin{tabular}{|c|c|c|c|c|c|c|c|c|}
\hline \multirow[t]{2}{*}{$\begin{array}{l}\text { Simulation } \\
\text { Time }\end{array}$} & \multicolumn{3}{|c|}{$\begin{array}{c}\text { Number of Packets Delivered at } \\
\text { Low Load }\end{array}$} & \multicolumn{3}{|c|}{$\begin{array}{c}\text { Number of Packets Delivered at } \\
\text { High Load }\end{array}$} & \multicolumn{2}{|c|}{ Latency } \\
\hline & Input & MCDR & TADR & Input & MCDR & TADR & MCDR & TADR \\
\hline 1 & 4.5 & 2 & 2 & 140 & 80 & 20 & 10 & 12 \\
\hline 2 & 10 & 6 & 4 & 110 & 60 & 50 & 11 & 16 \\
\hline 3 & 10 & 7 & 6 & 110 & 70 & 30 & 11 & 18 \\
\hline 4 & 16 & 14 & 10 & 120 & 80 & 25 & 11 & 18 \\
\hline 5 & 24 & 20 & 14 & 125 & 90 & 20 & 12 & 24 \\
\hline 6 & 24 & 21 & 14 & 125 & 85 & 40 & 12 & 26 \\
\hline 7 & 24 & 21 & 14 & 125 & 75 & 30 & 12 & 18 \\
\hline 8 & 25 & 21 & 14 & 125 & 80 & 35 & 12 & 15 \\
\hline 9 & 21 & 16 & 10 & 125 & 85 & 45 & 11 & 11 \\
\hline 10 & 18 & 12 & 6 & 115 & 90 & 10 & 11 & 18 \\
\hline 11 & 15 & 12 & 6 & 110 & 95 & 33 & 11 & 14 \\
\hline 12 & 15 & 8 & 4 & 110 & 95 & 38 & 11 & 14 \\
\hline
\end{tabular}

load condition over the performance of TADR. This increased performance is due to the greater loop reduction and scattering of traffic through the nodes which are placed at minimum distance to the sink in the network.

The Latency is depicted in Fig. 8 and the comparison values are tabulated in Table V. It is observed that the MCDR exhibits highly reduced Latency for both normal (1 to $4 \mathrm{~s}$ and 9 to $12 \mathrm{~s}$ ) and bursty traffic ( 3 to $8 \mathrm{~s}$ ). Whereas, the TADR exhibits high Latency for both normal and bursty traffic (1 to $12 \mathrm{~s}$ ). Thus, there is tremendous decrease in Latency of about $76.5 \%$ in MCDR when compared to TADR. This is due to cumulative 
effect of the parameters in consideration viz; minimum depth, maximum residual energy and Queue Length within the Threshold value of each node during traffic scattering.

Table VI lists with the number of packets delivered to the sink through different number of hops in MCDR and TADR. Fig. 9 shows that the MCDR exhibits higher rate of packet delivery through both minimum and maximum number of intermediate hops compared to the TADR. It is observed that the packet delivery rate is decreasing in TADR with increased number of hops (between the range of 30 to 90 hop distribution). Our protocol MCDR maintain better packet delivery rate irrespective of number of hops due to the fair queue length maintenance and minimum congestion rate at each node.

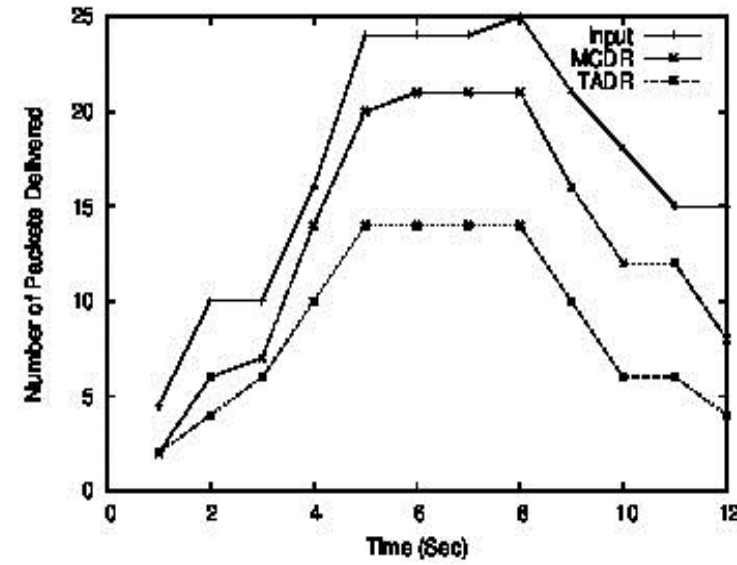

Figure 6 - Packet Delivery Rate at Low Load

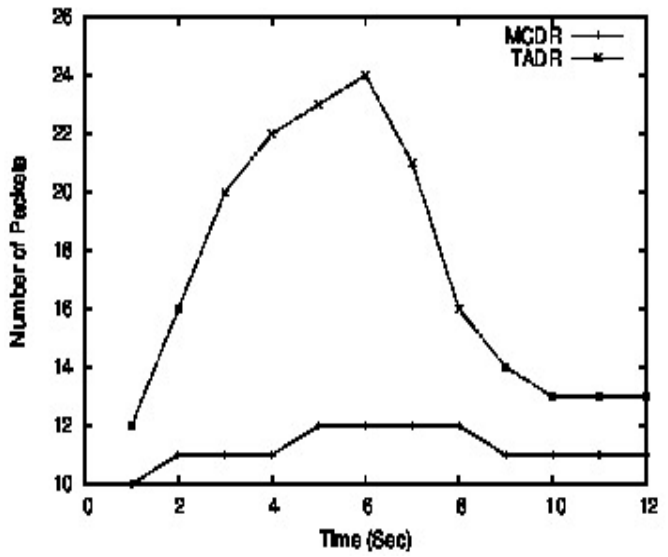

Figure - 8 Latency

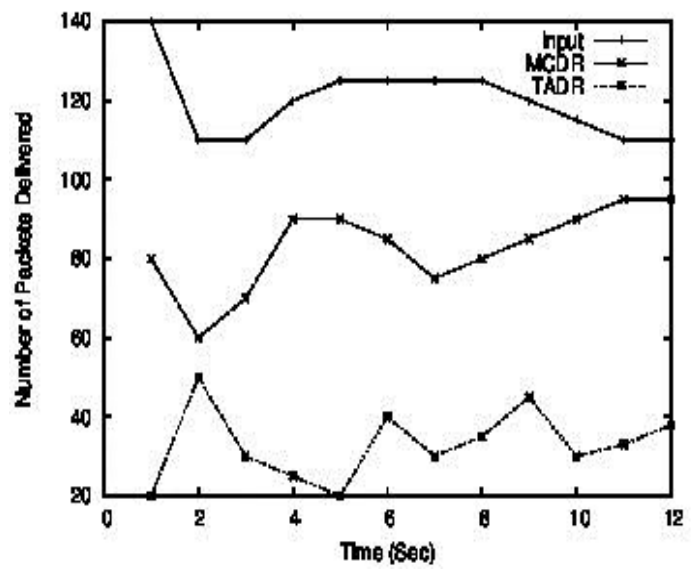

Figure 7 - Packet Delivery Rate at High Load

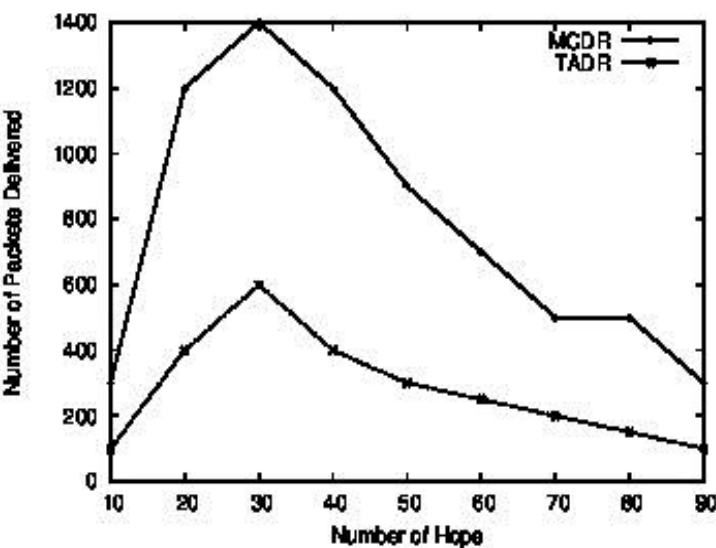

Figure -9 Hop Distributions

Table VI - Hop Distributions

\begin{tabular}{|l|l|l|}
\hline \multirow{2}{*}{ Number of Hops } & \multicolumn{2}{|l|}{ Number of Packets Delivered } \\
\cline { 2 - 3 } & MCDR & TADR \\
\hline 10 & 300 & 100 \\
\hline 20 & 1200 & 1000 \\
\hline 30 & 1400 & 1200 \\
\hline 40 & 1200 & 1000 \\
\hline 50 & 900 & 700 \\
\hline 60 & 700 & 500 \\
\hline 70 & 500 & 400 \\
\hline 80 & 500 & 300 \\
\hline 90 & 300 & 100 \\
\hline
\end{tabular}

VI. Conclusions

Congestion mitigation is one of the major issues in WSNs due to scarce resources. Thus, it is necessary to maximize the utilization of these resources to improve the overall network performance. In WSNs, congestion mainly affects the network throughput and in turn, it sluggishes the network performance. Our proposed algorithm MCDR effectively mitigates congestion by considering the parameters such as minimum Queue Length, the depth, the distance and maximum residual energy of each node while scattering the traffic 
towards the sink from the congested area. Improved network throughput is achieved by maintaining the minimum Congestion Rate due to fair Queue Length at each node in the network. The looping problem has been drastically reduced by selection of each node that is based on the minimum distance to scatter the traffic towards the sink. The reduction in looping results in lower Latency and minimizes energy utilization. The results of our proposed algorithm shows that improved network throughput and packet delivery rate for both high and low load conditions and also fulfils the fidelity requirement of different applications. Further, this work can be extended to support fidelity requirements of strict delay sensitive applications.

\section{References}

\section{Journal Papers:}

[1] Y. Yi and S. Shakkottai, "Hop-by-Hop Congestion Control over a Wireless Multi-Hop Network," In IEEE/ACM Transactions on Networking, vol. 15, no. 1, pp. 133-144, Feb. 2007.

[2] J. B. Helonde, V.Wadhai, V. Deshpande, and S. Sutar, "EDCAM: Early Detection Congestion Avoidance Mechanism for Wireless Sensor Network," In International Journal of Computer Applications, vol. 7, no. 2, pp. 11-14, Sep. 2010

[3] K. K. Sharma, H. Singh, and R. B. Patel "A Hop by Hop Congestion Control Protocol to Mitigate Traffic Contention in Wireless Sensor Networks," In International Journal of Computer Theory and Engineering, vol. 2, no. 6, pp. 986-991, Dec. 2010.

[4] S. Chen and N. Yang, "Congestion Avoidance Based on Lightweight Buffer Management in Sensor Networks," In IEEE Transactions Parallel and Distributed Systems, vol. 17, no. 9, pp. 934-946, Sep. 2006.

[5] J. Kang, Y. Zhang, and B. Nath, "TARA: Topology-Aware Resource Adaptation to Alleviate Congestion in Sensor Networks," In IEEE Transactions Parallel and Distributed Systems, vol. 18, no. 7, pp. 919-931, July 2007.

[6] R. Kumar, R. Crepaldi, H. Rowaihy, A.F. Harris, G. Cao, M. Zorzi, and T.F.L. Porta, "Mitigating Performance Degradation in Congested Sensor Networks," In IEEE Transactions Mobile Computing, vol. 7, no. 6, pp. 682-697, June 2008.

[7] N. Patwari, A.O. Hero III, M. Perkins, N. Correal, and R.J. O’Dea, "Relative Location Estimation in Wireless Sensor Networks," In IEEE Transactions Signal Processing, vol. 51, no. 8, pp. 2137-2148, Aug. 2003.

[8] J. Teo, Y. Ha, and C. Tham, "Interference-Minimized Multipath Routing with Congestion Control in Wireless Sensor Network for High-Rate Streaming, ” In IEEE Transactions Mobile Computing, vol. 7, no. 9, pp. 1124-1137, Sept. 2008.

[9] C. Wang, B. Li, K. Sohraby, M. Daneshmand, and Y. Hu, "Upstream Congestion Control in Wireless Sensor Networks through Cross-Layer Optimization," In IEEE Journal Selected Areas in Communication, vol. 25, no. 4, pp. 786-795, May 2007.

[10] Fengyuan Ren, Tao He, Sajal K. Das, and Chuang Lin, "Traffic-Aware Dynamic Routing to Alleviate Congestion in Wireless Sensor Networks", In IEEE Transactions on Parallel and Distributed Systems, vol. 22, no. 9, pp. 1585-1599, Sep. 2011.

\section{Proceedings Papers:}

[11] M. M. Monowar, Md. O. Rahman, and C. S. Hong, "Multipath Congestion Control for Heterogeneous Traffic in Wireless Sensor Network," In proceedings of $10^{\text {th }}$ International Conference on Advanced Communication Technology, ICACT, vol. 3, pp. 17111715, Feb. 2008

[12] M. M. Bhuiyan, I. Gondal, and J. Kamruzzaman, “CAM: Congestion Avoidance and Mitigation in Wireless Sensor Networks," In proceedings of IEEE Vehicular Technology Conference (VTC 2010-Spring), pp. 1-5, May 2010.

[13] C.Y. Wan, S.B. Eisenman, and A.T. Campbell, "CODA: Congestion Detection and Avoidance in Sensor Networks," In Proceedings of ACM International Conference Embedded Networked Sensor Systems (SenSys '03), pp. 266-279, 2003.

[14] Y. Sankarasubramaniam, O. Akan, and I. Akyildiz, "ESRT: Event to Sink Reliable Transport in Wireless Sensor Networks," In Proeedings of ACM Inernational Symonium Mobile Ad Hoc Networking and Computing (MobiHoc '03), vol. 13, no. 5, pp. 10031016, Oct. 2005.

[15] Mohammad Z. Ahmad and Damla Turgut, "Congestion Avoidance and Fairness in Wireless Sensor Networks," In proceedings of IEEE GLOBECOM, pp. 1-6, 2008.

[16] B. Scheuermann, C. Lochert, and M. Mauve, "Implicit Hop-by-Hop Congestion Control in Wireless Multihop Networks," In proceedings of Ad Hoc Networks, vol. 6, no. 2, pp. 260-286, April 2008.

[17] T. He, J. Stankovic, C. Lu, and T. Abdelzaher, "SPEED: A Stateless Protocol for Real-Time Communication in Sensor Networks," In Proceedings of IEEE International Conference Distributed Computing Systems (ICDCS ’03), pp. 46-55, May 2003.

[18] C. Wang, K. Sohraby, V. Lawrence, B. Li, and Y. Hu," Priority-Based Congestion Control in Wireless Sensor Networks," In proceedings of IEEE International Conference on Sensor Networks, 2006.

[19] C. Wan, S. Eisenman, A. Campbell, and J. Crowcroft, "Siphon: Over-Load Traffic Management using Multi-Radio Virtual Sinks in Sensor Networks," In Proceedings of ACM International Conference Embedded Networked Sensor Systems (SenSys '05), pp. 116$129,2005$.

[20] A. Basu, A. Lin, and S. Ramanathan, "Routing Using Potentials: A Dynamic Traffic-Aware Routing Algorithm," In Proceedings of ACM SIGCOMM, pp. 37-48, 2003.

[21] B. Hull, K. Jamieson, and H. Balakrishnan, "Mitigating Congestion in Wireless Sensor Networks," In Proceedings of International Conference Embedded Networked Sensor Systems (SenSys '04), pp. 134-147, 2004.

[22] J. Kang, B. Nath, Y. Zhang, and S. Yu, "Adaptive Resource Control Scheme to Alleviate Congestion in Sensor Networks," In Proceedings of First Workshop Broadband Advanced Sensor Networks, 2004.

[23] J. Paek and R. Govindan, "RCRT: Rate-Controlled Reliable Transport for Wireless Sensor Networks," In Proceedings of ACM International Conference Embedded Networked Sensor Systems (SenSys '07), 2007.

[24] A. Papadoulos and J.A. Mccann, "Towards the Design of an Energy-Efficient, Location-Aware Routing Protocol for Mobile, AdHoc Sensor Networks," In Proceedings of 15th International Workshop Database and Expert Systems Applications, pp. 705-709, 2004.

[25] P. P. Pham and S. Perreau, "Performance Analysis of Reactive Shortest Path and Multipath Routing Mechanism with Load Balance," In Proceedings of IEEE INFOCOM, vol. 1, pp. 251-259, Apr. 2003.

[26] L. Popa, C. Raiciu, I. Stoica, and D.S. Rosenblum, "Reducing Congestion Effects in Wireless Networks by Multipath Routing," In Proceedings of IEEE International Conference Network Protocols (ICNP '06) ,pp. 96-105, Nov. 2006.

[27] S. Rangwala, R. Gummadi, R. Govindan, and K. Psounis, "Interference-Aware Fair Rate Control in Wireless Sensor Networks," In Proceedings of ACM SIGCOMM, pp. 63-74, 2006.

[28] M.H. Yaghmaee and D.A. Adjeroh, "Priority-Based Rate Control for Service Differentiation and Congestion Control in Wireless Multimedia Sensor Networks," In Proceedings of Computer Networks, vol. 53, no. 11, pp. 1798-1811, 2009. 


\section{Authors}

B M Thippeswamy is an Assistant Professor and Head in the Department of Computer Science and Engineering at Sambhram Institute of Technology, Bangalore, India. $\mathrm{He}$ obtained his B.E in Computer Science and Engineering from Mysore University and M.E degrees in Computer Science and Engineering from Bangalore University, Bangalore. He is presently pursuing his Ph.D programme in the area of Wireless Sensor Networks in JNTU Anantpur, India. His research interest is in the area of Wireless Sensor Networks.

Raghavendra $\mathbf{M}$ is an Assistant Professor in the of Computer Science and Engineering at Siddaganga Institute of Technology, Tumkur, India. He received his Bachelors degree in Computer Science and Engineering from Visvesvaraya Technological University and Master of Engineering in the Department of Computer Science and Engineering at University Visvesvaraya College of Engineering, Bangalore University, Bangalore, India. His research interest is in the area of Sensor Networks.

Reshma $\mathbf{S}$ is an Assistant Professor in the Department of Computer Science and Engineering at Sambhram Institute of Technology, Bangalore, India. She received her Bachelors degree in Computer Science and Engineering from Visvesvaraya Technological University and Master of Technology from Visvesvaraya Technological University, Regional Center, Bangalore. Her research interest is in the area of Wireless Sensor Networks.

Shaila $\mathbf{K}$ is an Professor and Head in the Department of Electronics and Communication Engineering at Vivekananda Institute of Technology, Bangalore, India. She obtained her B.E in Electronics and M.E degrees in Electronics and Communication Engineering, and $\mathrm{Ph} . \mathrm{D}$ degree from Bangalore University, Bangalore. Her research interests are in the area of Sensor Networks, Adhoc Networks and Image Processing.

Venugopal $\mathbf{K} \mathbf{R}$ is currently the Principal, University Visvesvaraya College of Engineering, Bangalore University, Bangalore. He obtained his Bachelor of Engineering from University Visvesvaraya College of Engineering. He received his Masters degree in Computer Science and Automation from Indian Institute of Science Bangalore. He was awarded Ph.D. in Economics from Bangalore University and Ph.D. in Computer Science from Indian Institute of Technology, Madras. He has a distinguished academic career and has degrees in Electronics, Economics, Law, Business Finance, Public Relations, Communications, Industrial Relations, Computer Science and Journalism. He was a
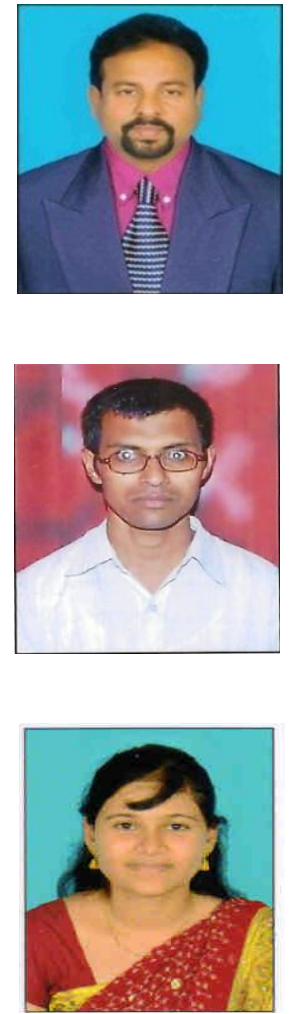
postdoctoral research scholar at University of Southern California, USA. He has authored and edited 39 books on Computer Science and Economics, which include Petrodollar and the World Economy, C Aptitude, Mastering C, Microprocessor Programming, Mastering C++ and Digital Circuits and Systems etc.. During his three and half decades of service at University Visveraya College of Engineerng. He has over 300 research papers to his credit. His research interests include Computer Networks, Wireless Sensor Networks, Parallel and Distributed Systems, Digital Signal Processing and Data Mining.

S S Iyengar S S Iyengar is currently Ryder Professor, Florida International University, USA. He was Roy Paul Daniels Professor and Chairman of the Computer Science Department at Louisiana State University. He heads the Wireless Sensor Networks Laboratory and the Robotics Research Laboratory at LSU. He has been involved with research in High Performance Algorithms, Data Structures, Sensor Fusion and Intelligent Systems, since receiving his Ph.D degree in 1974 from MSU, USA. He is Fellow of IEEE and ACM. He has directed over 55 Ph.D students and 100 Post Graduate students, many
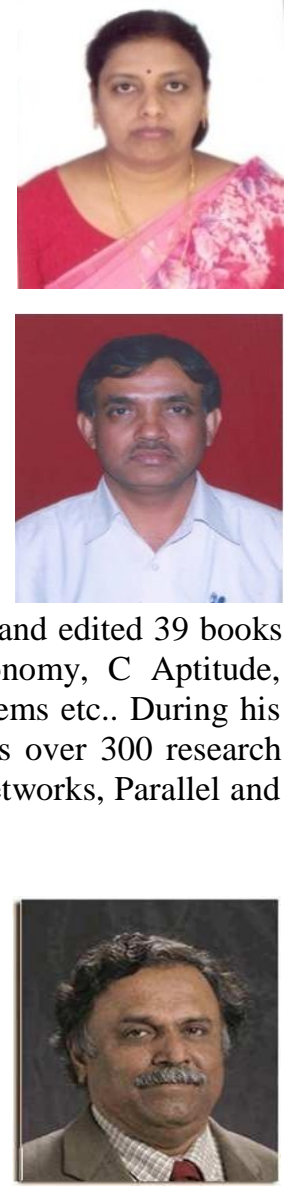
of whom are faculty at Major Universities worldwide or Scientists or Engineers at National Labs/Industries around the world. He has published more than 500 research papers and has authored/co-authored and edited 16 books. His books are published by John Wiley \& Sons, CRC Press, Prentice Hall, Springer Verlag, IEEE Computer Society Press etc.. One of his books titled Introduction to Parallel Algorithms has been translated to Chinese.

L M Patnaik is currently Honorary Professor, Indian Institute of Science, Bangalore, India. He was a Vice Chancellor, Defense Institute of Advanced Technology, Pune, India and was a Professor since 1986 with the Department of Computer Science and Automation, Indian Institute of Science, Bangalore. During the past 35 years of his service at the Institute he has over 700 research publications in refereed International Journals and refereed International Conference Proceedings. He is a Fellow of all the four leading Science and Engineering Academies in India; Fellow of the IEEE and the Academy of Science for the Developing World. He has received twenty national and international

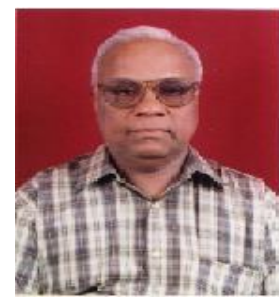
awards; notable among them is the IEEE Technical Achievement Award for his significant contributions to High Performance Computing and Soft Computing. His areas of research interest have been Parallel and Distributed Computing, Mobile Computing, CAD, Soft Computing and Computational Neuroscience. 